\title{
Evidence for coronal activity cycles on 61 Cygni A and B
}

\author{
A. Hempelmann ${ }^{1}$, J. H. M. M. Schmitt ${ }^{1}$, S. L. Baliunas ${ }^{2}$, and R. A. Donahue ${ }^{2}$ \\ 1 Universität Hamburg, Hamburger Sternwarte, Gojenbergsweg 112, 21029 Hamburg, Germany \\ e-mail: ahempelmann@hs .uni-hamburg.de \\ 2 Harvard-Smithsonian Center for Astrophysics, 60 Garden Street, MS 15, Cambridge, MA 02138, USA
}

Received 2 May 2003 / Accepted 11 June 2003

\begin{abstract}
We investigate a four-and-one-half year time-series of ROSAT HRI pointed observations of 61 Cyg A and B and compare the X-ray light curves with the chromospheric Ca HK variability. The ROSAT sampling rate was two pointings per year and typical errors lie in the range of 5-10\%. The chromospheric cycles are well-known for both stars from the Mt. Wilson Ca HK survey. Although the time basis of our ROSAT observations is shorter than the 7-and 12-year cycles of components A and B, respectively, we find the long-term trend of coronal activity in close correlation with the chromospheric activity during the observation period, between 1993 and 1998. The chromospheric activity increased through maximum activity down to a minimum for component $\mathrm{A}$, and from maximum to minimum activity for component $\mathrm{B}$. The same behaviour is observed for the X-ray light curves but with much higher amplitudes by factors $2.5-3$. The remaining scatter observed around low-order regression curves of coronal activity is small. We conclude that both stars do show coronal cycles and that coronal cycles are the dominant source of variability for 61 Cygni.
\end{abstract}

Key words. stars: late-type - stars: activity - stars: coronae - stars: chromospheres

\section{Introduction}

Magnetically induced activity of the Sun manifests itself in a variety of phenomena, the oldest and most prominent of which is the eleven year sunspot cycle. The eleven year cycle is also visible in many other activity indicators, in particular, in those originating in the chromosphere or corona. Examples of chromospheric activity tracers that clearly vary in phase with the sunspot cycle are the $\mathrm{Ca}$ II $\mathrm{H}$ and $\mathrm{K}$ line core emission (Wilson 1978; White \& Livingston 1981; Keil \& Worden 1984), MgII h+k and Lyman $\alpha$ (Chandra et al. 1995). Such activity indicators can contain some fraction of non-magnetic origin (as observed in Ca II H+K, Schrijver 1983) lowering the amplitude of any variations. However, the X-ray emission of late type stars is believed to be of pure magnetic origin (Stępień \& Ulmschneider 1989). Hence, an activity cycle should be most prominent in X-rays. Indeed, the Sun varies in its X-ray luminosity by more than one order of magnitude, indicating a strong coronal activity cycle in phase with the solar spot cycle (Kreplin 1970). One therefore expects that other stars should also exhibit large cyclical variations in their X-ray output. However, the question is whether coronal cycles are observable on stars where typically no more than a few snapshot observations per year can be made with X-ray observatories. In the case where there are other strong sources of variability,

Send offprint requests to: A. Hempelmann, e-mail: ahempelmann@hs .uni-hamburg.de such as flaring, active region emerging and decaying or rotational modulation, a coronal cycle may be invisible.

For stars rotating below the mass-dependent saturation limit, the level of coronal activity of cool stars is determined by stellar rotation (Pizzolato et al. 2003). Hempelmann et al. (1996) investigated the scatter around a relation between X-ray stellar surface flux $\left(F_{\mathrm{X}}\right)$ and Rossby number (the Rossby number is the rotation scaled by the convective turnover time) for a sample of stars with clear chromospheric cycles as determined from the Mt. Wilson Ca HK survey (Baliunas et al. 1995). They correlated the residuals to that relation with the known phases of chromospheric cycles, and found (with a confidence of $2 \sigma$ ) that the X-ray residuals depend on the cycle phase of chromospheric activity and claimed this as a hint of coronal cycles inherent the data. One of those stars investigated was the cool star binary 61 Cygni. 61 Cygni is a well-studied nearby binary consisting of two late-type stars of spectral types K5V and K7V with an angular separation of only 26 arcsec. Consequently it was observed with the ROSAT High Resolution Imager (HRI). The result of these observations is subject of this paper.

\section{Observations}

The ROSAT HRI observations of 61 Cygni were started in the middle of 1993 and stopped at the end of the ROSAT operations in 1997 . We obtained a total number of 9 pointings with typical exposure times of a few kiloseconds giving typical Poisson $1 \sigma$ 
Table 1. ROSAT HRI Observations of $61 \mathrm{Cyg}$ A and B.

\begin{tabular}{cccc}
\hline \hline $\begin{array}{c}\text { Time } \\
\text { year }\end{array}$ & $\begin{array}{c}\text { HRI count-rate } \\
\text { cts/s }\end{array}$ & $\begin{array}{c}\text { Errror } \\
\text { cts/s }\end{array}$ & $\begin{array}{c}L_{\mathrm{X}} \\
\mathrm{erg} / \mathrm{s}\end{array}$ \\
\hline \multicolumn{4}{c}{ Component A } \\
\hline 1993.44 & 0.061 & 0.005 & $2.145 \times 10^{27}$ \\
1994.86 & 0.079 & 0.008 & 2.795 \\
1994.86 & 0.071 & 0.003 & 2.506 \\
1995.44 & 0.087 & 0.004 & 3.075 \\
1995.95 & 0.063 & 0.003 & 2.224 \\
1996.39 & 0.063 & 0.003 & 2.210 \\
1996.87 & 0.048 & 0.003 & 1.690 \\
1997.34 & 0.031 & 0.003 & 1.096 \\
1997.84 & 0.046 & 0.005 & 1.602 \\
\hline \multicolumn{4}{c}{ Component B } \\
\hline 1993.44 & 0.028 & 0.003 & $9.778 \times 10^{26}$ \\
1994.86 & 0.026 & 0.005 & 9.042 \\
1994.86 & 0.024 & 0.002 & 8.581 \\
1995.44 & 0.026 & 0.002 & 9.239 \\
1995.95 & 0.016 & 0.002 & 5.587 \\
1996.39 & 0.013 & 0.002 & 4.563 \\
1996.87 & 0.014 & 0.002 & 4.870 \\
1997.34 & 0.014 & 0.002 & 5.017 \\
1997.84 & 0.011 & 0.002 & 3.789 \\
\hline
\end{tabular}

errors of 5-10\% for each flux measurement. Concerning variations on time-scales of years, the typical sampling of half a year should be sufficient.

The two stellar components are clearly resolved by the HRI field of view, making a standard reduction process possible: corrections for dead time, vignetting and background subtraction. A count-rate to flux conversion factor of $2.4 \times$ $10^{-11} \mathrm{ergs} / \mathrm{cm}^{2} / \mathrm{counts}$ and a distance of $3.48 \mathrm{pc}$ (Perryman et al. 1997) are used to calculate X-ray luminosities. Their uncertainties will not affect our results because we do not investigate absolute brightness but its variability. The data are given in Table 1; they are also available from the ROSAT data archive and from the ROSAT Database for Nearby X-ray and extreme UV emitting Stars, NEXXUS (Schmitt \& Liefke 2003).

\section{Results}

\subsection{Cygni $A$}

The component A of 61 Cygni exhibits a clear cyclic behaviour in its chromospheric activity (Fig. 1). The cycle period was determined to be 7.3 years (Baliunas et al. 1995). Figure 3 shows a section of the available Ca HK time-series spanning a bit more than the four-and-one-half years of ROSAT observations. It includes both ascending and descending parts around a maximum of activity in 1995. Exactly this temporal behaviour is also visible in the ROSAT X-ray light curve (Fig. 4). Furthermore, the maximal coronal activity appears exactly at the time of maximum chromospheric activity. This strongly suggests that the cycles of chromospheric and coronal activities are in phase.

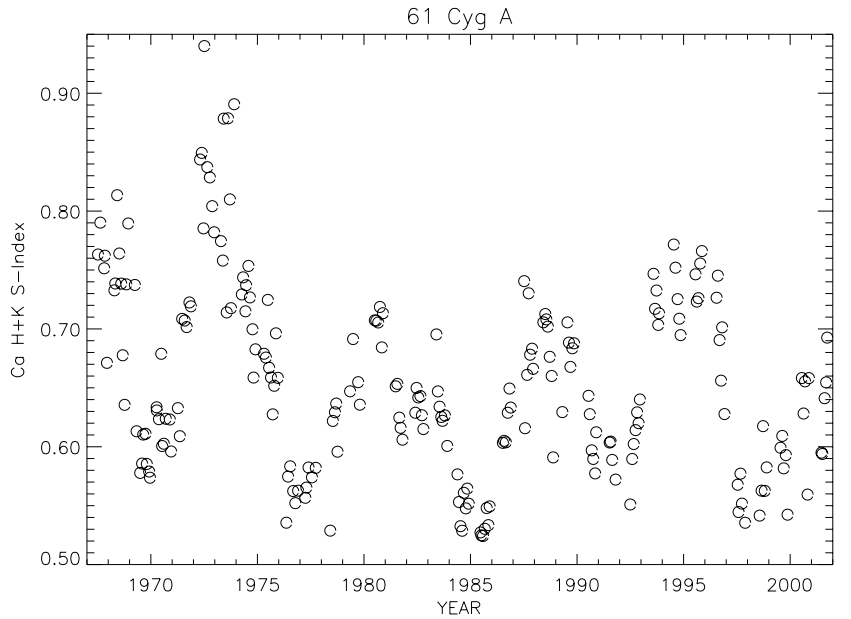

Fig. 1. Time-series of the Ca II $\mathrm{H}+\mathrm{K}$ Mt. Wilson $S$-Index of 61 Cygni A.

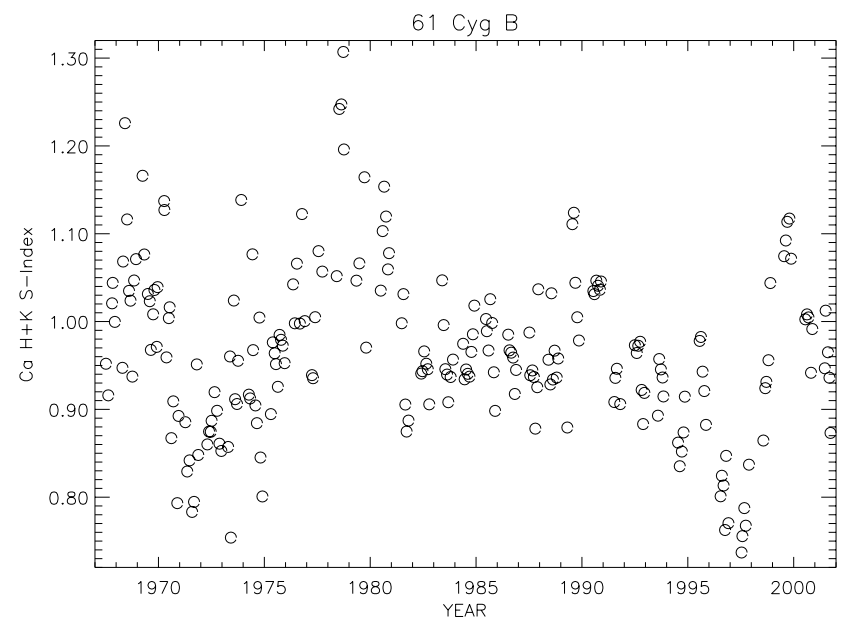

Fig. 2. As Fig. 1 but for the stellar component B.

Unfortunately, more than half of the ROSAT data points lie in the seasonal gaps of the Ca HK observations which hampers a direct correlation analysis. In order to find a suitable $\mathrm{Ca} \mathrm{H}+\mathrm{K} S$ value for a comparison with a ROSAT observation, we therefore attempted three methods. First we took the $\mathrm{Ca} \mathrm{HK}$ observation that is closest to a ROSAT data point. Second, we made a linear interpolation between the two neighboured points and, third, we fit the whole curve by a polynomial (regression curve in Fig. 3) and compared the value of the regression curve at the time of ROSAT observation with the corresponding X-ray measurement. The results are given in Table 2 . The correlation between $L_{\mathrm{X}}$ and the interpolated $S$ value (derived by help of the third method) is demonstrated in Fig. 5.

\subsection{Cygni B}

The time interval of our ROSAT observations cover only a relatively small fraction of the 11.7-year Ca HK cycle determined by Baliunas et al. (1995). Fortunately, this time interval coincides with the most pronounced variation between 1993 and 1998 of the Ca HK time-series. During those four and onehalf years, a strong and more or less linear decrease of $\mathrm{Ca} \mathrm{HK}$ 


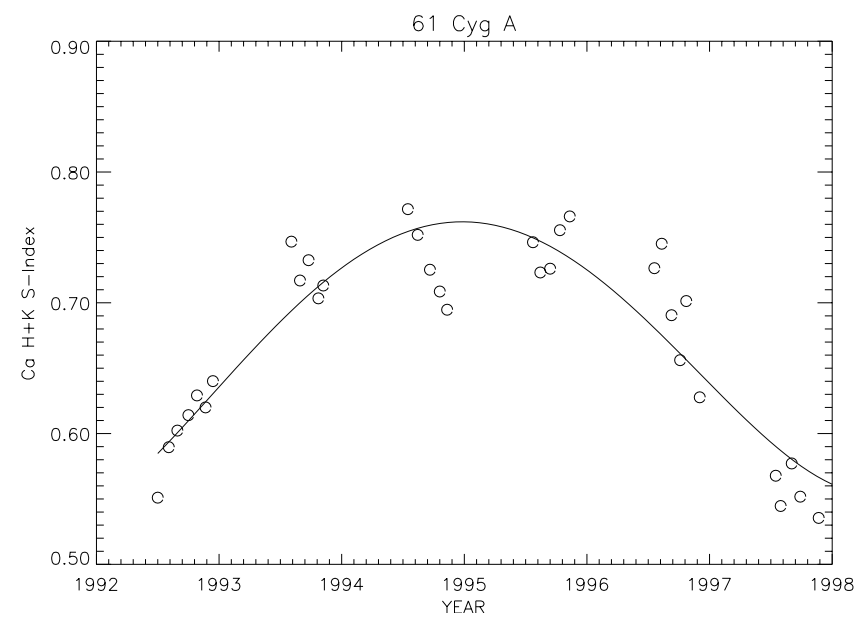

Fig. 3. Detail of Fig. 1 concerning the period of ROSAT HRI observations. The curve is a fourth order polynomial fit.

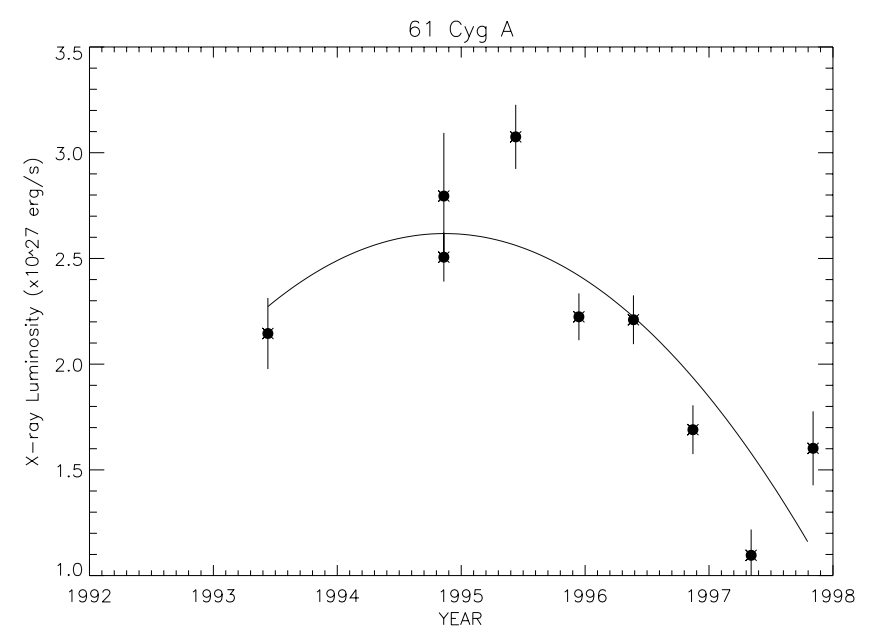

Fig. 4. Time-series of the ROSAT HRI pointed observations of stellar component A. The curve is a second order polyxnomial fit.

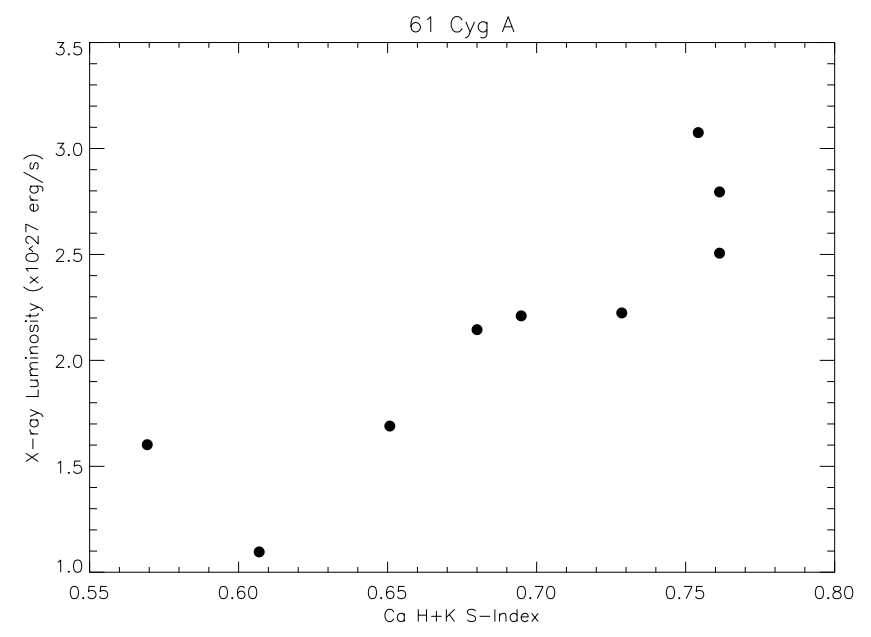

Fig. 5. Linear correlation between $L_{\mathrm{x}}$ and Ca HK $S$ for stellar component A. The $S$ value is taken from the fit in Fig. 3 at the time of ROSAT HRI observation.

activity was observed (Fig. 2). However, as Fig. 6 shows this global linear trend is overlayed with short-term fluctuations.
Table 2. Linear correlation coefficients between X-ray and Ca HK activities of 61 Cygni stellar components A and B.

\begin{tabular}{ccc}
\hline \hline Method of comparison with Ca H+K $S$ & A & B \\
\hline Closest $S$ data point & 0.58 & 0.73 \\
Interpolation between $S$ neighbours & 0.68 & 0.74 \\
$S$-curve fit value & 0.93 & 0.88 \\
\hline
\end{tabular}

Figure 7 demonstrates that there is also an almost linear trend visible in the ROSAT data. Furthermore, it might be possible that the coronal activity follows the Ca HK short-term fluctuations, too. But this idea is rather speculative because most of the X-ray observations lie in seasonal gaps of the $\mathrm{Ca} \mathrm{HK} \mathrm{ob-}$ servations. Hence, we have restricted our investigation to the global trends in Figs. 6 and 7. Table 2 and Fig. 8 demonstrate a good correlation between chromospheric and coronal activity of the stellar component $\mathrm{B}$, too.

\section{Discussion}

While component $\mathrm{A}$ is an excellent example of a cyclic star (Fig. 1), the long-term variations of component B do not appear as a regular sine curve. It looks rather chaotic with strong short-term fluctuations (Fig. 2). However, the close connections in the long-term trends depicted in Figs. 3, 4 and respectively, 6,7 which find their expression in the high correlation cofficients of Table 2, demonstrate that coronal cycles are visible in the X-ray time series of both stellar components. The relatively small residual scatter around the regression curves of Figs. 4 and 7 suggests further that such cyclic activity dominates over other sources of X-ray variability, in contrast to the $\mathrm{Ca}$ HK observations, where short-term fluctuations on a timescale of approximately one year are strong, at least in case of stellar component B.

The amplitude of the long-term X-ray variability is factors 2.5-3 larger than the factors 1.1-1.4 which were observed for the Ca HK cycles between 1993 and 1998. (However, the measure $S$ of Ca HK activity is contaminated by constant sources like photospheric contributions, which will damp the observed amplitudes.)

Schmitt \& Liefke (2003) compared the ROSAT PSPC RASS count-rates with count-rates of ROSAT PSPC pointed observations of a sample of Gliese catalogue stars. There the scatter around unity is interpreted as caused by stellar variability. They find a factor of four between maximium and minimum deviations. Thus the cycle amplitudes of 61 Cygni A and B lie well inside this scatter band.

There are a few stars of the Schmitt \& Liefke (2003) sample which lie outside the scatter band mentioned above. All these stars are known flare stars.

\section{Conclusions}

We have investigated two long-term time series of X-ray and Ca HK observations of two stars with known chromospheric activity cycles. Although the time basis of four-and-one-half years of ROSAT pointing observations is too short to cover 


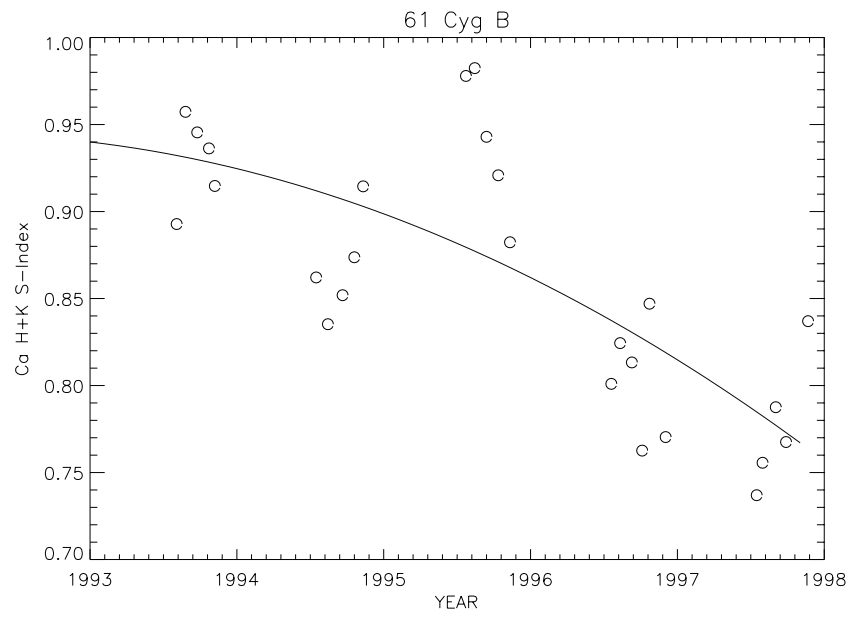

Fig. 6. Detail of Fig. 2 concerning the period of ROSAT HRI observations. The curve is a second order polyxnomial fit.

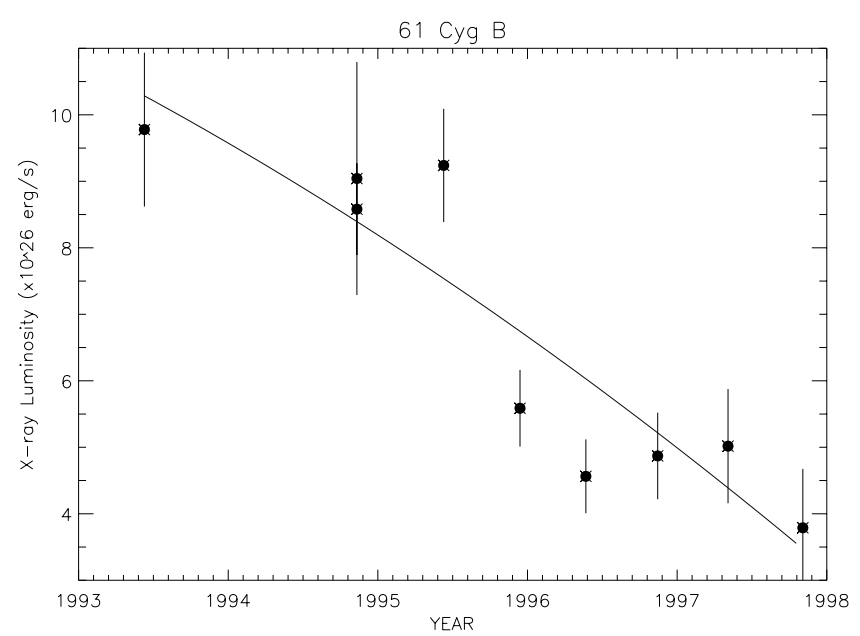

Fig. 7. Time-series of the ROSAT HRI pointed observations of stellar component $\mathrm{B}$. The curve is a second order polyxnomial fit.

completely the seven-year cycle of $61 \mathrm{Cyg}$ A or the twelve-year cycle of $61 \mathrm{Cyg}$ B, the timing of the X-ray observations in both cases covered phases of maximum chromospheric variability. Our ROSAT HRI observations follow closely the observed trends in the chromospheric cycles but with much higher amplitudes than observed for the chromospheric cycles. This suggests strongly that, following the solar analogy, coronal cycles do exist and that they are observable. Because the individual scatter around our low order regression curves of coronal activity is smaller by a factor of three in comparison with its large amplitude, we can further conclude that other sources

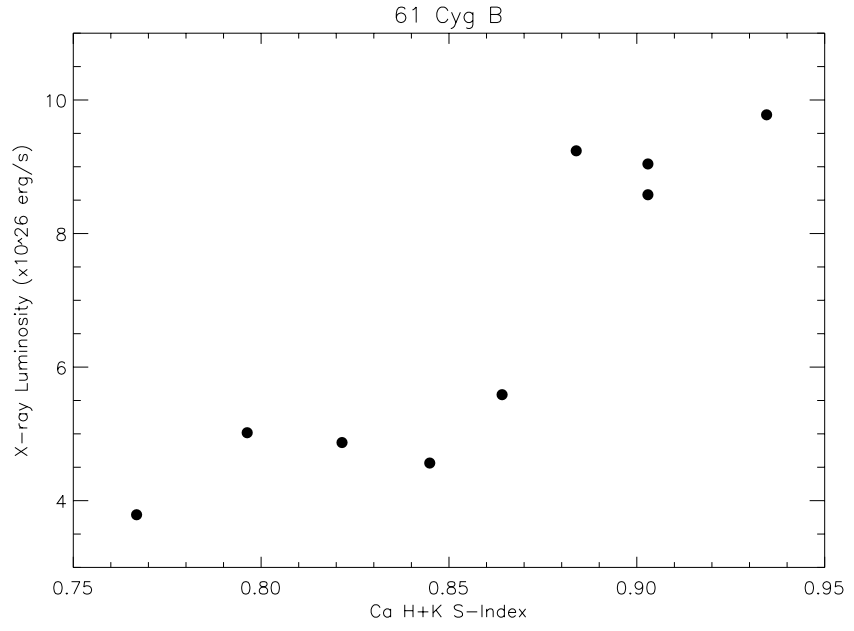

Fig. 8. Linear correlation between $L_{\mathrm{x}}$ and Ca HK $S$ for stellar component B. The $S$ value is taken from the fit in Fig. 6 at the time of ROSAT HRI observation.

of variability such as rotational modulation or individual evolution of active regions play only a minor role. Thus, coronal cycles (and maybe occasional flaring) seem to be the main sources of coronal variability of 61 Cygni.

Acknowledgements. This work was supported by funds from the National Aeronautics and Space Administration (NAG5-7635) and the Air Force Office of Scientific Research (AF 49620-02-1-0194).

\section{References}

Baliunas, S. L., Donahue, R. A., Soon, W. H., et al. 1995, ApJ, 438, 269

Chandra, S., Lean, J. L., White, O. R., et al. 1995, Geoph. Res. Lett., 22,2481

Hempelmann, A., Schmitt, J. H. M. M., \& Stępień, K. 1996, A\&A, 305,284

Keil, S. L., \& Worden, S. P. 1984, ApJ, 276, 766

Kreplin, R. W. 1970, Ann. Géophys., 26, 567

Perryman, M. A. C., Lindegren, L., Kovalevsky, J., et al. 1997, A\&A, 323, L49

Pizzolato, N., Maggio, A., Micela, G., Sciortino, S., \& Ventura, P. 2003, A\&A, 397, 147

Schmitt, J. H. M. M., \& Liefke, C. 2003, A\&A, in press

Schrijver, C. J. 1983, A\&A, 127, 289

Stępień, K., \& Ulmschneider, P. 1989, A\&A, 216, 139

White, O. R., \& Livingston, W. C. 1981, ApJ, 249, 798

Wilson, O. C. 1978, ApJ, 226, 379 